\title{
Un'osservazione sull'andamento annuo dei valori orari di grandezze geofisiche a controllo solare
}

\author{
P. DOMINICI
}

1. Esiste una abbastanza vasta categoria di grandezze geofisiche il cui valore dipende, in maniera più o meno diretta, anche dall'altezza del Sole sull'orizzonte (grandezze a controllo solare), e che cioè possono essere rappresentate da una funzione del tipo:

$$
y=f\left(\ldots, x_{i}, \ldots, \chi\right)
$$

essendo $\chi$ la distanza zenitale del Sole e le $x_{i}$ altre variabili, diverse da $\chi$. Poichè $\chi$ è legata all'angolo orario $\omega$ del Sole dalla ben nota relazione:

$$
\cos \chi=\operatorname{sen} \varphi \operatorname{sen} \delta+\cos \varphi \cos \delta \cos \omega,
$$

dove $\varphi$ è la latitudine del luogo d'osservazione e $\delta$ è la declinazione solare, la $y$ puó riguardarsi come funzione delle $x_{i}$, di $\varphi, \delta$ e $\omega$ :

$$
y=f^{*}\left(\ldots, x_{i}, \ldots, \chi, \delta, \omega\right)
$$

Se le rilevazioni di $y$ fossero effettuate sistematicamente con un programma orario riferito a $\omega$, ciò̀ al tempo solare vero, l'andamento nel corso dell'anno del valore $\bar{y}$ relativo al valore particolare $\bar{\omega}$ di $\omega$ risulterebbe descritto da una funzione del tipo:

$$
y=f^{*}(\ldots, \ldots, \ldots, \varphi, \delta, \bar{\omega})
$$

nella quale, posto che il luogo d'osservazione rimanga sempre il medesimo, comparirebbero come variabili indipendenti solo le $x_{i}$ e $\delta$.

Se invece, come si fa abitualmente, il programma delle rilevazioni di $y$ viene stabilito con riferimento al tempo civile della stazione d'osservazione $P$, tale andamento assume la forma:

$$
\bar{y}=f^{*}\left(\ldots, x_{i}, \ldots, \varphi, \delta,\left[\bar{\omega}+\omega_{12}\right]\right),
$$


nella quale compare una nuova variabile, l'angolo orario $\omega_{12}$ del Sole al mezzogriorno civile di $P$, che misura la differenza tra il tempo solare vero e il tempo civile in $P$.

Com'è noto, ̀̀

$$
\omega_{12}=\varepsilon+\Lambda
$$

dove $\varepsilon$, che gli astronomi chiamano equazione del tempo, ̀̀ la differenza, variabile nel corso dell'anno, tra il tempo solare vero e il tempo solare medio e $\Lambda$ è la longitudinale di $P$ rispetto al meridiano centrale del fuso orario al quale $P$ appartiene, positiva o negativa secondo che $P$ si trovi a Est oppure a Ovest di tale meridiano. Naturalmente l'andamento nel corso dell'anno di $\omega_{12}$ ripete, a meno della costante addittiva $\Lambda$, quello di $\varepsilon$ (v. fig. 1).

La [5] può essere scritta, in forma puramente simbolica, come segue:

$$
\bar{y}=\bar{y} F\left(\omega_{12}\right)
$$

L'andamento annuo dei valori orari della $\bar{y}$ rilevati in $P, \bar{y}$, differisce pertanto da quello, $y$, peculiare della $y$ medesima per una grandezza, $F\left(\omega_{12}\right)$, che, dipendendo dalla scelta del riferimento temporale, è di natura puramente convenzionale.

Nel presente lavoro ci proponiamo di discutere, in via del tutto indicativa, l'entità dell'errore, o, se si preferisce, della "distorsione di forma ", introdotto dalla funzione $F\left(\omega_{12}\right)$, precedentemente definita, nell'andamento annuo dei valori orari di una grandezza del tipo [1]: precisamente, sull'andamento annuo dei valori meridiani di densità elettronica massima in uno strato ionosferico.

2. Consideriamo uno strato ionosferico nel quale l'equilibrio elettronico sia governato da un'equazione della forma seguente:

$$
\frac{\partial N}{\partial t}=q_{0} \cos \chi-\beta N
$$

dove $N$ è il numero di elettroni liberi per $\mathrm{cm}^{3}, q_{0}$ il numero di elettroni liberi prodotti per $\mathrm{cm}^{3}$ e per secondo nello strato allorchè il Sole culmina allo zenit (cioè allorquando è $\omega=0$ ) e $\beta$ una quantità, fisicamente interpretabile come coefficiente di attaccamento tra elettroni liberi e molecole circostanti, che supporremo costante. Osserviamo incidentalmente che la [8] può assumersi, almeno in prima approssimazione, a rappresentare le condizioni esistenti nella regione F2 della ionosfera.

Poniamo:

$$
N(t)=N_{0} \nu(t)
$$


dove $N_{0}$ è il valore di $N$ per $\chi=0$. Risulta poi conveniente, in virtù aella [2], passare dalla variabile $t$, in secondi, alla variabile $\omega$, in radianti (I), sicchè scriveremo la [8] nella forma:

$$
\begin{gathered}
\frac{\partial y}{\partial \omega}=\frac{{ }_{x \mathrm{u}}^{\pi}}{N_{0}}(A+B \cos \omega)-a B \nu \\
a=86^{\prime} 400 / 2 \pi=1,3710^{4} \\
A=\operatorname{sen} \varphi \operatorname{sen} \delta \\
B=\cos \varphi \cos \delta .
\end{gathered}
$$

A norma della [9] la funzione adimensionata $\nu(\omega)$ deve quindi soddisfare la condizione:

Posto:

$$
\left\{\begin{array}{l}
v\left({ }_{0}\right)=1 \\
A+B=1
\end{array}\right.
$$

$$
\left(\frac{\partial \nu}{\partial \omega}\right)_{\chi=0}=C
$$

e:

$$
C=\frac{a q_{0}}{\omega_{0}}-a \beta,
$$

e la [10] diviene:

$$
\frac{\partial \nu}{\partial \omega}=(a B+C)(A+B \cos \omega)-a B \nu .
$$

Riguardando la $v$ come funzione della sola variabile $\omega$, la soluzione generale della [13] risulta la seguente:

$\left\{\begin{array}{l}\nu(\omega)=(a \beta+C) A+(a \beta+C) \frac{R}{1+\ddot{\omega}^{\circ} \hat{\gamma}^{\circ}}(\operatorname{sen} \omega+a \beta \cos \omega)+\gamma \exp [-a \beta \omega] \\ \gamma=\text { costante }\end{array}\right.$

Assumiamo, per semplicità, $a \beta=1$ : ciò equivale ad assumere per $\beta$ il valore $7,3 \cdot 10^{-5}$, valore del resto fisicamente verosimile. Con tale posizione, la soluzione particolare della [13] che soddisfa la [10'] è la seguente:

$$
\begin{gathered}
\nu(\omega)=(1+C) A+\frac{(1+C) B}{2}(\operatorname{sen} \omega+\cos \omega)+ \\
+\frac{1-C-(1+C) A}{2} \exp [-\omega]
\end{gathered}
$$


L'andamento annuo dei valori di $v$ relativi all'istante del passaggio del Sole al meridiano della stazione d'osservazione $P$, cioè per $\omega=0$, è espresso dalla funzione:

$$
\nu\left({ }^{\circ}\right)=\frac{(1+C)(A+B)+1-C}{2},
$$

che è del tipo [4].

L'andamento annuo dei valori di $\nu$ al mezzogiorno civile di $P$ è invece rappresentato dalla funzione:

$$
\begin{gathered}
v\left(\omega_{12}\right)=(1+C) A+\frac{(1+C) R}{2}\left(\operatorname{sen} \omega_{12}+\cos \omega_{12}\right)+ \\
+\frac{1-C-(1+C) A}{2} \exp \left[-\omega_{12}\right]
\end{gathered}
$$

che è del tipo [5].

Come appare dalla fig. $1, \varepsilon$ è sempre abbastanza piccolo, non superando, in valore assoluto, 0,07 radianti; $\Lambda$, da parte sua, non supera,

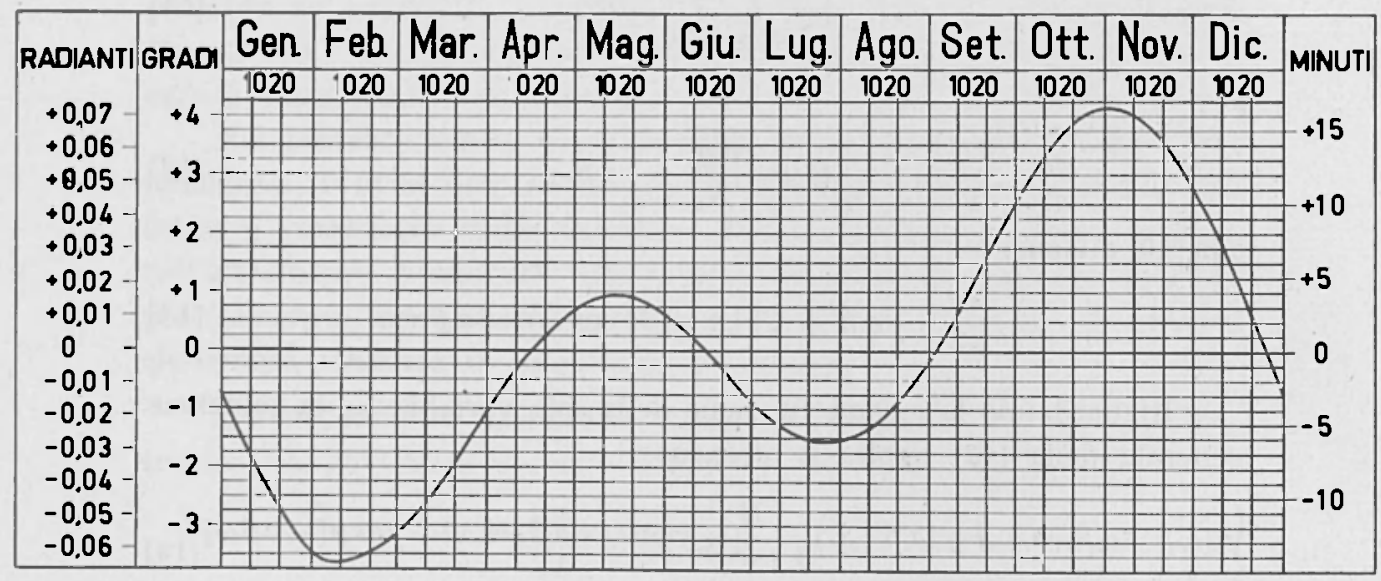

Fig. I - Andamento nel corso dell'anno dell'equazione del tempo.

in valore assoluto, $7^{\circ} 30^{\prime}$, pari a circa 0,13 radianti. Complessivamente (II) $\omega_{12}$ si mantiene, in valore assoluto, tanto piccolo da potersi scrivere, con buona approssimazione:

$$
\left\{\begin{aligned}
\operatorname{sen} \omega_{12}+\cos \omega_{12} & \cong 1+\omega_{12}-\frac{\omega_{12}{ }^{2}}{2} \\
\exp \left[-\omega_{12}\right] & \cong 1-\omega_{12}+\frac{\omega_{12}^{2}}{2}
\end{aligned}\right.
$$


sicchè la [17] può scriversi:

$$
\begin{aligned}
& v\left(\omega_{12}\right)=\frac{(1+C)(A+B)+1-C}{2}+ \\
& +\left(\omega_{12}-\frac{\omega_{12}^{2}}{\hat{z}}\right) \frac{(1+C)(A+B)-1+C}{2} .
\end{aligned}
$$

Confrontando le [16] e [19] si ha infine:

$$
\frac{\nu\left(\omega_{12}\right)}{\nu\left({ }_{0}\right)}=1+\left(\omega_{12}-\frac{\omega_{12}^{2}}{2}\right) \frac{(1+C)(A+B)-1+C}{(1+C)(A+B)+1-C} .
$$

Se:

$$
|C| \ll 1
$$

l'errore percentuale $\Delta v$ introdotto da $\omega_{12}$ nei valori $\nu(o)$ vale:

$$
\Delta v=100\left(\omega_{12} \ldots \frac{\omega_{12}^{2}}{2}\right) \frac{A+B-1}{A+B+1} .
$$

Se invece non vale la condizione [21], in luogo della [22] dovremo considerare la seguente relazione:

$$
\Delta \nu^{*}=\Delta \nu+100\left(\omega_{12}-\frac{\omega_{12}{ }^{2}}{2}\right) C .
$$

Nella fig. 2 è riportato l'andamento della quantità $\Delta v$ tra le latitudini $67^{\circ} 30^{\prime} \mathrm{S}$ e $67^{\circ} 30^{\prime} \mathrm{N}$ per stazioni al centro del proprio fuso orario $(\Lambda=0)$ e al limite Est $\left(\Lambda=7^{\circ} 30^{\prime}\right)$ e Ovest $\left(\Lambda=-7^{\circ} 30^{\prime}\right)$ del fuso stesso, il 15 di ogni mese.

Indichiamo con $f_{0_{0}}$ la frequenza critica che si misurerebbe in una data stazione al mezzogiorno solare, con $f_{0.12}$ quella che si deduce dagli ionogrammi registrati al mezzogiorno civile. Ricordando che è:

si ha dalla [20]:

$$
N=1,2410^{-8} f_{0}^{2}
$$

$$
\frac{f_{0,12}}{f_{0,0}}=\left(1+\frac{\Delta v}{100}\right)^{1 / 2}
$$

ovvero, per la relativa piccolezza del termine $\frac{\Delta \nu}{100}$ :

$$
\frac{f_{012}}{f_{0,0}}=1+\frac{\Delta v}{50} \text {. }
$$

Poichè si deve ritenere che, in generale, le frequenze critiche ionosferiche siano misurate con una precisione non inferiore a $\pm 1 \%$, l'effetto qui considerato non può a rigore essere trascurato appena $\Delta v$ superi il va- 
lore \pm 2 , il che, come si vede dalla fig. 2 , accade frequentemente alle medie e alte latitudini per stazioni in posizione eccentrica nel proprio fuso orario.

Nella fig. 3 è riportato l'andamento di $\nu\left(\omega_{12}\right)$ relativo a due stazioni a latitudine $45^{\circ} \mathrm{N}$. geograficamente assai vicine ma appartenenti a due fusi orari diversi: una di tali stazioni (curva $a$ in fig. 3) risulta pertanto

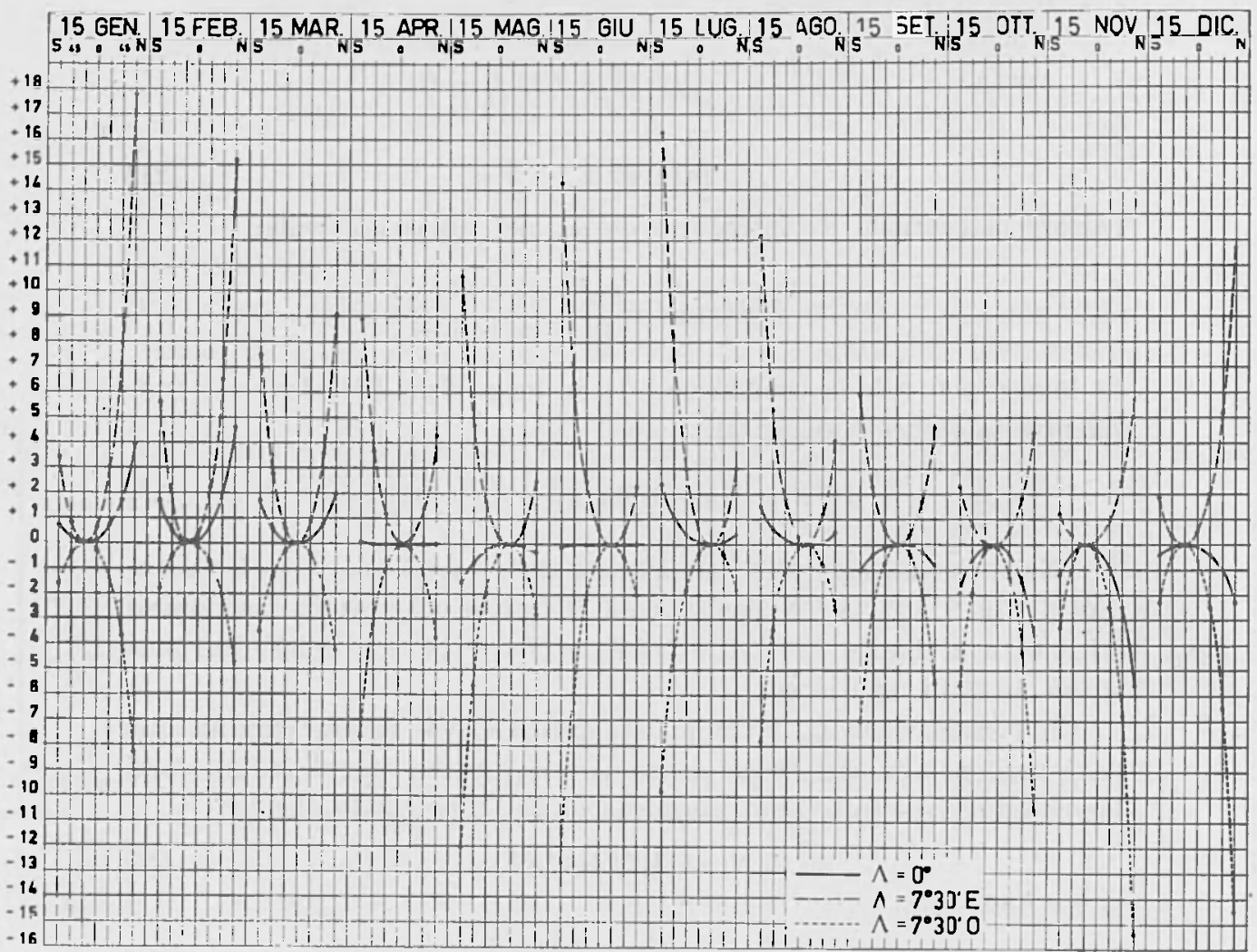

Fig. 2

tutta spostata a Est, l'altra (curva $b$ in fig. 3) tutta spostata a Ovest nel proprio fuso orario. Nella stessa fig. 3 la curva $c$ rappresenta la differenza percentuale $\Delta f_{0,12}$ delle frequenze critiche $f_{012}$ misurate nella prima stazione rispetto a quelle misurate nella seconda: osserviamo che tale differenza sarebbe, naturalmente, nulla se le due stazioni operassero con lo stesso programma orario. La curva tratteggiata, intermedia tra le 
curve $a$ e $b$, indica l'andamento della quantità $\nu\left({ }_{0}\right)$ per le due stazioni considerate.

3. Le conclusioni alle quali siamo giunti nella discussione testè conclusa su un caso molto particolare portano a ritenere che, in generale, debba porsi attenzione all'effetto qui considerato ogniqualvolta si debbano considerare andamenti annui di grandezze geofisiche a controllo

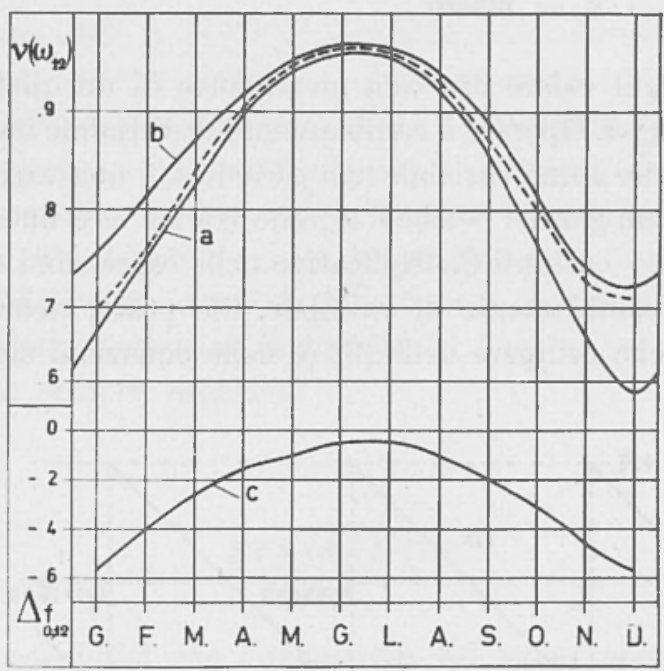

Fig. 3

solare i cui valori orari siano riferiti al tempo civile delle stazioni d'osservazione o al tempo universale. Spetta naturalmente allo sperimentatore, sulla base della natura della grandezza investigata e della precisione intrinseca dei dati d'osservazione, decidere sull'opportunità o meno di introdurre un'opportuna correzione sui dati rilevati.

La questione ci sembra tuttavia che abbia non trascurabile importanza per le grandezze ionosferiche, per le quali il controllo solare è molto stretto; particolarmente sensibili all'effetto considerato dovrebbero essere le frequenze critiche della regione F2, che, com'è noto, presentano variazioni orarie molto cospicue. 


\section{APPENDICE}

(I) Il legame tra $t$ e $\omega$ è espresso dalla seguente relazione:

$$
\left\{\begin{array}{l}
t=\frac{86^{\prime} 400}{2 \pi}(\omega+\pi)+k 86^{\prime} 400 \\
k=\text { intero }
\end{array}\right.
$$

ovvero, detto $t_{0}$ il valore di $t$ alla mezzanotte di un qualunque giorno, dal grafico di fig. 4. Operare il cambiamento di variabile indicato significa pertanto sostituire a una variabile non periodica, $t$, una variabile, $\omega$, periodica di periodo un giorno; poichè il legame tra $t \mathrm{e} \omega$ è lineare, si vengono a introdurre solo costanti moltiplicative nelle espressioni differenziali in cui si opera il cambiamento di variabile. Ciò posto, ricorderemo che la funzione $N(t)$ che compare nella [8] (e nelle equazioni similari che pos-

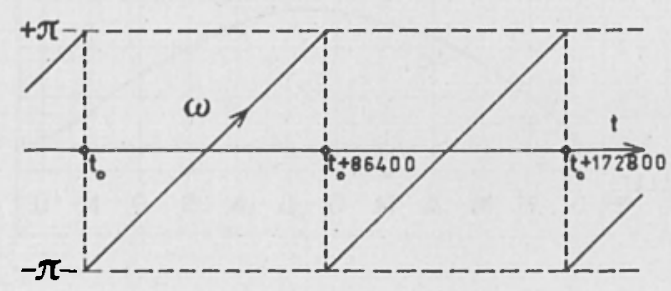

Fig. 4

sono scriversi considerando diversi e più complessi meccanismi di equilibrio elettronico nella ionosfera) è una funzione pseudoperiodica, di pseudoperiodo un giorno: la $N(t)$ si ripete infatti, a meno di trascurabili differenze, in giorni successivi ma, naturalmente non si conserva inalterata nel corso di un intero anno. Dato che la variabile $t$ non è periodica, per rispecchiare tale realtà fisica si impone usualmente alla soluzione generale della [8] di essere periodica di periodo un giorno: il che, come ben si comprende, non è rigorosamente esatto. Operando il cambiamento di variabile sopra indicato, la pseudoperiodicità della funzione rimane soddisfatta per la natura stessa della nuova variabile, e l'unica condizione postulabile per la funzione, cioè il soddisfacimento di una conveniente condizione iniziale, rimane di natura puramente analitica. 
(II) Ricordiamo che, per convenzione (*), uno ionogramma s'intende rilevato nell'istante in cui la frequenza della ionosonda è di $3 \mathrm{MHz}$. Detto $\tau$ l'intervallo di tempo impiegato dalla ionosonda per passare da $3 \mathrm{MHz}$ a $8 \div 15 \mathrm{MHz}$, valori propri delle più alte fra le frequenze critiche ionosferiche, la [6] andrebbe a rigore modificata come segue:

$$
\omega_{12}=\varepsilon+\Lambda+\tau,
$$

nella quale espressione $\tau$ s'intende naturalmente misurato nella stessa unità nella quale sono misurati $\varepsilon$ e $A$. La quantità $\tau$ puó ritenersi del tutto trascurabile per le ionosonde " veloci ", che impiegano da 7 secondi a 2 minuti per esplorare l'usuale campo di frequenza da 1 a $20 \mathrm{MHz}$ : può invece giungere a valori notevoli nelle ionosonde "lente", nelle quali la durata di rilevazione dello ionogramma va da 4 a 10 minuti. Comunque, dato che le ionosonde attualmente in uso rilevano gli ionogrammi per valori crescenti di frequenza, $\tau$, quando non è trascuravile, è una quantità sempre negativa.

\section{RIASSUNTO}

Esiste la possibilità che l'andamento dei valori orari di grandezz" geofisiche a controllo solare (che dipendano ciò̀ anche dalla distanza zenitale $\chi$ del Sole) possa essere alterato dal fatto che $i$ valori di tali grandezze siano rilevati con un programma riferito al tempo civile della stazione d'osservazione anzichè al tempo solare vero. A titolo indicativo viene calcolata l'entità di tale "distorsione di forma " in un caso particolare, quello dei valori meridiani di densita elettronica massima $N$ di uno strato iono-

(*) IGY Instruction Manual, vol. I1I, part. I (Ionospheric Vertical Soundings), pag. 157.

\section{Sounding Schedules}

3. Timing. The reference time for ionospheric sounders is that of the zero meridian (UT), altough local standard meridian time, differing from zero meridian time by an integral number of hours, is more convenient and slrould be used in all reports. ... . The nominal time of a sounding is defined as the time when the ionosonde records the standard frequency $3 \mathrm{Mc}$. The nominal and schedule time of sounding should be equal within $0.5 \mathrm{~min}$. 
sferico nel quale l'equilibrio elettronico sia governato da un'equazione del tipo: $\frac{\partial N}{\partial t}-f(\chi)-\beta N$. Le conclusioni alle quali si perviene in questo caso particolare portano a ritenere che, in generale, si debba porre attenzione a tale effetto, massimamente per stazioni a media e alta latitudine in posizione eccentrica nel proprio fuso orario.

\section{$A B S T R A C T$}

There is a possibility that behaviour of hourly values of geophysical data depending from the zenith distance $\chi$ of the Sun may be altered by the fact that these values are computed with reference to the civil time of the observing station and not to the solar time. For the sake of an example, the amount of such "distortion of form". has been calculated for the particular case of the meridian values of the maximum electron density $N$ of a ionospheric layer in which the electronic balance, is given by an equation of the following type: $\frac{\partial N}{\partial t}=f(\chi)-\beta N$. The results $o b$ tained in this particular case allow us to think that this effecte must be, generally, into consideration, expecially for medium-and high-latitude stations whose position in their respective time zones is eccentric. 